\title{
Link budget analysis for satellite-based narrowband IoT systems ${ }^{\star}$
}

\author{
Oltjon Kodheli ${ }^{1}$, Nicola Maturo ${ }^{1}$, Stefano Andrenacci ${ }^{2}$, Symeon $_{\text {Chatzinotas }}{ }^{1}$, \\ and Frank Zimmer ${ }^{2}$ \\ 1 Snt - Interdisciplinary Centre for Security, Reliability and Trust, University of \\ Luxembourg, 29 Avenue J.F. Kennedy,Luxembourg City L-1855, Luxembourg \\ ${ }^{2}$ SES, Château de Betzdorf, Rue Pierre Werner, 6815 Betzdorf, Luxembourg
}

\begin{abstract}
Low-power wide-area networks (LPWAN) have been rapidly gaining ground in recent years, triggered by their capability to satisfy important market segments. Narrowband Internet of Things (NB-IoT) is one of the most appealing LPWAN technologies, foreseen to play an important role in the fifth generation mobile communication $(5 \mathrm{G})$ network. In order to guarantee a worldwide coverage to the low-cost devices distributed all over the globe, satellite connectivity is a key asset due to their large footprint on Earth, especially in remote areas where the investment towards a terrestrial infrastructure is not justified. However, such terrestrial networks aiming at deploying satellite systems either as an integrated part of it or a stand-alone solution, would require a careful and detailed analysis covering several aspects and all the layers of communication. In this paper, we demonstrate the link budgets of a satellite-based NB-IoT system under different parameters, providing some simulation results as a benchmark for further study. In addition, we analyze and discuss the impact that different power budgets would have in important features of the NB-IoT network, such as delay, capacity and device battery life.
\end{abstract}

Keywords: 5G · NB-IoT $\cdot$ Link Budget Analysis · Satellite Communication - Spectral Efficiency

\section{Introduction}

In the last years, the Internet of things (IoT) has drawn a great deal of research attention, both from academia and industry, due to the impact it is expected to have in the global economic processes and the quality of everyday life [9, $18,22]$. The number of IoT devices generating and exchanging information with each-other is estimated to be three times as high as the global population by 2020 [12]. In order to satisfy this tremendous market demand, the 3rd Generation Partnership Project (3GPP) introduced the narrowband Internet of things

* This work was supported by the Luxembourg National Research Fund (FNR) under Industrial Fellowship Scheme with industrial partner SES S.A., project title "Communication algorithms for end-to-end satellite-IoT (SATIOT)", grant FNR12526592. 
(NB-IoT) standard [5], which is foreseen to play an important role in the fifth generation mobile communication $(5 \mathrm{G})$ network. A crucial key performance indicator (KPI) of this technology is to guarantee a worldwide connectivity to the low-cost IoT devices distributed all over the globe. However, in many cases the terrestrial infrastructure does not exist and it has a very high deployment cost. For this reason, the satellite connectivity is considered to be a very attractive solution in such areas in order to complement and extend the coverage of the terrestrial network. Several contributions have studied such systems, showing the fundamental features and the role of the satellites in the 5G IoT communications [11] [10][21]. Moreover, in our previous works we studied an NB-IoT over a LEO satellite system, providing a solution to reduce the high differential Doppler shift [15][16].

Together with other technical challenges and considerations, link budget is an important aspect worth analyzing for satellite-based NB-IoT networks, motivated by the following reasons. On the one hand, even though the link budget is already well-studied for terrestrial NB-IoT through several contributions [17][19], a new analysis is needed since the constraints in a satellite system are different with respect to a terrestrial one. More specifically, because of the presence of the satellite, a power constraint will be present both in the downlink (forward link) and uplink (return link) case. Indeed, one of the main challenges in a satellite communication system is where to get the power from, which in a terrestrial system this is not an issue. Solar power is the most likely source of energy to be used in space, imposing a significant limitation in closing the communication link, due to the difficulty of generating large power quantities onboard the satellite. On the other hand, 3GPP recently completed a study item in $5 \mathrm{G}$ air interface to support non-terrestrial networks (NTN)[4], where the link budgets for different satellite altitudes and frequency bands were shown. However a specific analysis targeting only the NB-IoT is necessary, due to the particular technical peculiarities of this technology. In the literature, some research works already exist, studying the coverage extension of NB-IoT through LEO satellite [14][13]. Nevertheless, due to the recent development of the NB-IoT standard with improved capabilities and the new 3GPP agreements for the satellite link design in NTN 5G air interface, an updated and more detailed link budget analysis is of utmost importance.

As a result, in this paper, we analyze the link budget for a satellite-based NB-IoT network, having as a baseline the latest 3GPP specifications regarding the system level parameters. Additionally, we provide some simulation results as a benchmark for further study and discuss the impact that different power budget levels at the receiver would have in the overall system design.

The remainder of the paper is structured as follows. In the next section, we give a brief overview of the NB-IoT technology. Section 3 is devoted to the link budget analysis. Section 4 presents the impact of the link budget in the overall system design and the concluding remarks are given in Section 5. 
To be presented in the 18th International Conference on Ad Hoc Networks and Wireless

(AdHoc-Now 2019), Luxembourg, 1-3 October 2019.

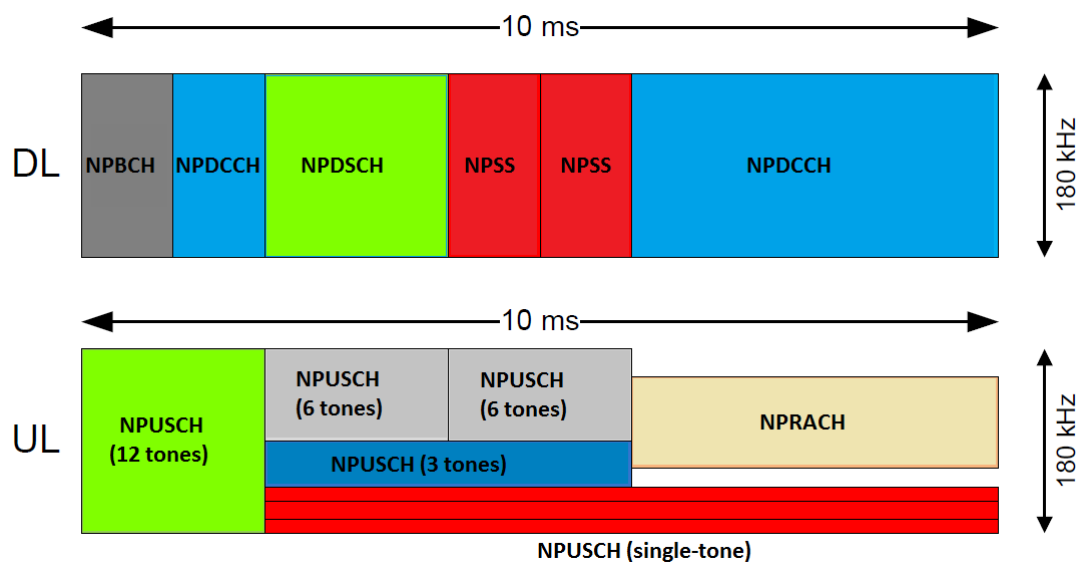

Fig. 1. NB-IoT Radio Frame Design [20]

\section{NB-IoT overview}

The aim of this section is to recall only some important information related to the NB-IoT technology, which will be useful for a better understanding of the other sections of the paper.

\subsection{General Features}

The following features have been introduced in LTE Release 13 for NB-IoT [8]: a) Support of massive number of low-throughput devices (around 52547) within a cell coverage; b) Ultra-low complexity and low-cost devices; c) Improved power consumption efficiency to allow battery life of more than ten years. The NB-IoT system requires a bandwidth of $180 \mathrm{kHz}$ in order to operate. This also corresponds to a physical resource block (PRB) in LTE, since they are designed to coexist. Based on where the NB-IoT carrier is placed within the LTE carrier there can be identified three operational modes: in-band, guard-band and stand-alone. The downlink transmission uses the conventional Orthogonal Frequency Division Multiple Access (OFDMA) with $15 \mathrm{kHz}$ subcarrier spacing (SCS), whereas the uplink transmission uses the Single Carrier Frequency Division Multiple Access (SC-FDMA) with 3,75 SCS or $15 \mathrm{kHz}$ SCS. For the uplink, both single-tone (ST) and multi-tone (MT) transmissions (i.e., 3, 6, and 12 subcarriers) are supported.

\subsection{PHY channels and signals}

There are three downlink physical channels in NB-IoT. The narrowband physical broadcast channel $(\mathrm{NPBCH})$ sends the information related to the cell and network configuration. The narrowband physical downlink control channel (NPD$\mathrm{CCH}$ ) sends all the control signals regarding important procedures such as paging, random access, and data transmission. The narrowband physical downlink 
To be presented in the 18th International Conference on Ad Hoc Networks and Wireless (AdHoc-Now 2019), Luxembourg, 1-3 October 2019.

shared channel (NPDSCH) is responsible for sending the data and control information, acknowledgment (ACK) or negative ACK (NACK) of a Hybrid Automatic Repeat reQuest (HARQ) process, from the base station to the users.

Only two channels exist in the uplink. The narrowband physical uplink shared channel (NPUSCH) is used for sending user data transmission from the users to the base station or control information (ACK/NACK). Narrowband physical random access channel (NPRACH) is used by the users to access the network and synchronize for data transmission.

Figure 1 demonstrates how these channels can be scheduled in downlink and uplink in the time-frequency resources of the NB-IoT radio frame. It can be noted that in the uplink, since there exist different transmission modes, using less subcarriers in the frequency domain would result in a longer channel in the time domain. Besides, some resources in the uplink frame should be reserved for the NPRACH in order to allow other users to access the network and synchronize for uplink data transmission. Contrarily, in the downlink transmission, the channels are multiplexed in time, since one channel occupies all the available frequency resources of $180 \mathrm{kHz}$. An important aspect worth mentioning here is that the transmission can be configured with different modulation and coding schemes (MCS), causing this way different performance gain, device energy consumption, capacity and coverage levels. Last but not least, an important feature of NB-IoT is the use of the repetition code. This means that each channel can be repeated multiple times in time in order to improve the signal to noise ratio (SNR), thus extending the coverage. Together with the MCS selection, the number of repetition used would determine the overall system performance.

\section{$3 \quad$ Satellite Link Budget Formula}

In a telecommunication system, the link budget analysis is done to relate the power at the receiver with regard to the power at the transmitter, accounting for signal gains and losses in the propagation medium. By neglecting the interference, the link budget between a transmitter and a receiver in free space is given by the carrier power over noise density $(\mathrm{C} / \mathrm{N})$ as a function of other system and link parameters. The general formula of the link budget, accounting for all the gains and looses in the propagation medium from transmitter to receiver and neglecting the interference, is given as follows [3]:

$$
\begin{aligned}
\frac{C}{N}(d B)=E I R P(d B W)+\frac{G_{r}}{T}(d B i / K) & -F S P L(d B)-A_{\text {loss }}(d B)-A d_{\text {loss }}(d B) \\
& -K\left(\frac{d B W / K}{H z}\right)-10 \cdot \log _{10}(B W)
\end{aligned}
$$

Let us now clarify each of the above parameters one by one.

- EIRP is the effective isotopic radiated power of the transmitting antenna and can be calculated as: 
To be presented in the 18th International Conference on Ad Hoc Networks and Wireless (AdHoc-Now 2019), Luxembourg, 1-3 October 2019.

$$
E I R P=10 \cdot \log _{10}\left(G_{T} P_{T}\right)
$$

where $P_{T}$ is the transmitting antenna power and $G_{T}$ is the gain.

- $G_{r} / T$ is the figure of merit at the receiver having antenna gain $G_{r}$ and equivalent system temperature $T$ derived by the following:

$$
\frac{G_{r}}{T}=G_{r}(d B i)-N F(d B)-10 \cdot \log _{10}\left(T_{o}+\left(T_{a}-T_{o}\right) \cdot 10^{-0.1 \cdot N F}\right)
$$

where $G_{r}$ is the gain of the receiving antenna, $N F$ represents the noise figure, $T_{o}$ is the ambient temperature and $T_{a}$ is the antenna temperature.

- FSPL is the free space path loss given by:

$$
F S P L=10 \cdot \log _{10}\left(\frac{4 \pi D}{c / f}\right)^{2}
$$

with carrier frequency $f$, speed of light $c$ and slant range $D$ expressed as:

$$
D=-R_{E} \cdot \sin (\alpha)+\sqrt{R_{E}^{2} \cdot \sin (\alpha)^{2}+h_{s}+2 \cdot R_{E} \cdot h_{s}}
$$

The slant range is the distance from the user device to the satellite and it can be noted from the formula that it is determined by the radius of Earth $R_{E}$, satellite elevation angle $\alpha$ and satellite altitude $h_{s}$.

- $A_{\text {loss }}$ and $A d_{\text {loss }}$ represent the atmospheric looses due to gases, rain fades etc., and additional looses due to feeder link.

- $B W$ is the communication bandwidth and $K$ is the Boltzman constant.

\subsection{Simulation Parameters and Results}

The goal of radio link design is to guarantee a reliable communication between a transmitter and receiver. In the context of NB-IoT systems, link reliability is evaluated through the block error rate (BLER) associated with the specific MCS, which depends on the available SNR. By utilizing equation 1, it is possible to calculate the SNR (or written as $\mathrm{C} / \mathrm{N}$ ) at the receiver under specific system parameters, both in the downlink and uplink transmission. The user terminal parameters are the ones defined in the NB-IoT standard for 3GPP Class 3 devices [1], whereas the link parameters can be taken from the $3 \mathrm{GPP}$ specification for $5 \mathrm{G}$ over NTN [3], summarized in Table 1. Moreover, we leave on purpose undefined the satellite parameters (EIRP in downlink and $G / T$ in uplink) because these are the ones that should be carefully designed before launching new satellites to support NB-IoT services or check whether the existing ones meet the power budget requirements. Changing these satellite parameters would directly affect the received SNR.

We use the link level performance results, shown in Appendix, to determine the required SNR values corresponding to a $10 \%$ BLER at the first HARQ transmission. Different MCS levels in NB-IoT can achieve different spectral efficiency 
To be presented in the 18th International Conference on Ad Hoc Networks and Wireless (AdHoc-Now 2019), Luxembourg, 1-3 October 2019.

Table 1. Link Budget Parameters [3].

\begin{tabular}{|c|c|c|}
\hline Link Parameters & Downlink & Uplink \\
\hline Carrier Frequency (GHz) & 2 & 2 \\
Bandwidth (kHz) & 180 & $3.75,15,45,90,180$ \\
Subcarrier Spacing (kHz) & 15 & $3.75,15$ \\
Satellite Altitude for LEO (km) & 600 & 600 \\
Satellite Altitude for GEO (km) & 35786 & 35786 \\
Minimum Elevation Angle (degree) & 30 & 30 \\
Atmospheric Loss LEO and GEO (dB) & 0.5 & 0.5 \\
Additional Loss LEO and GEO (dB) & 1 & 1 \\
Channel model & AWGN & AWGN \\
\hline Terminal Parameters & & $3 \mathrm{GPP}$ Class 3 \\
Terminal Type & $3 \mathrm{GPP}$ Class 3 & - \\
Antenna Type & 0 & - \\
Receiver Antenna Gain (dBi) & 9 & - \\
Terminal Noise Figure (dB) & 290 & - \\
Terminal Ambient Temperature (K) & 290 & 23 \\
Terminal Antenna Temperature (K) & - & 0 \\
Terminal Transmit Power (dBm) & - & Omnidirectional \\
Terminal Transmit Antenna gain (dBi) & -
\end{tabular}

as shown in Table 3. Therefore, combining these results with the link budget formula in equation 1 , it is possible to obtain the spectral efficiency as a function of satellite EIRP for downlink case and G/T for uplink case, as illustrated in Figure 2, 3 and 4 . We have taken into account only Low Earth Orbit (LEO) and Geostationary (GEO) satellite, with the corresponding altitudes given in the Table 1, because these are the ones considered in the latest 3GPP studies.

It can be noted that, in the downlink case, in order to enable an NB-IoT system capable of achieving the highest possible spectral efficiency, it is needed a minimum EIRP of $25 \mathrm{dBW}$ for a LEO satellite at $600 \mathrm{~km}$ altitude and $57 \mathrm{dBW}$ for a GEO satellite at $35786 \mathrm{~km}$ altitude. Having a higher EIRP at the satellite does not give any further gain since these are the NB-IoT system limitations. On the other hand, in case these EIRP values are not guaranteed, still it is possible to close the link, but with lower spectral efficiency.

In the uplink transmission, the analysis is a bit more complex due to the existence of several transmission modes. For a 12-carriers transmission mode it is required a minimum $\mathrm{G} / \mathrm{T}$ of $-2 \mathrm{~dB} / \mathrm{K}$ for a $\mathrm{LEO}$ satellite and $28 \mathrm{~dB} / \mathrm{K}$ for a GEO satellite. In case of lower values of $\mathrm{G} / \mathrm{T}$, the link can still be closed by reaching the peak spectral efficiency, but by using the other transmission modes (e.g. 1,3 or 6 subcarriers) for the SC-FDMA signal. However, even though the peak spectral efficiency is guaranteed by means of different transmission modes, this will have an impact on the overall system design, as we will analyze in the following section. 
To be presented in the 18th International Conference on Ad Hoc Networks and Wireless (AdHoc-Now 2019), Luxembourg, 1-3 October 2019.

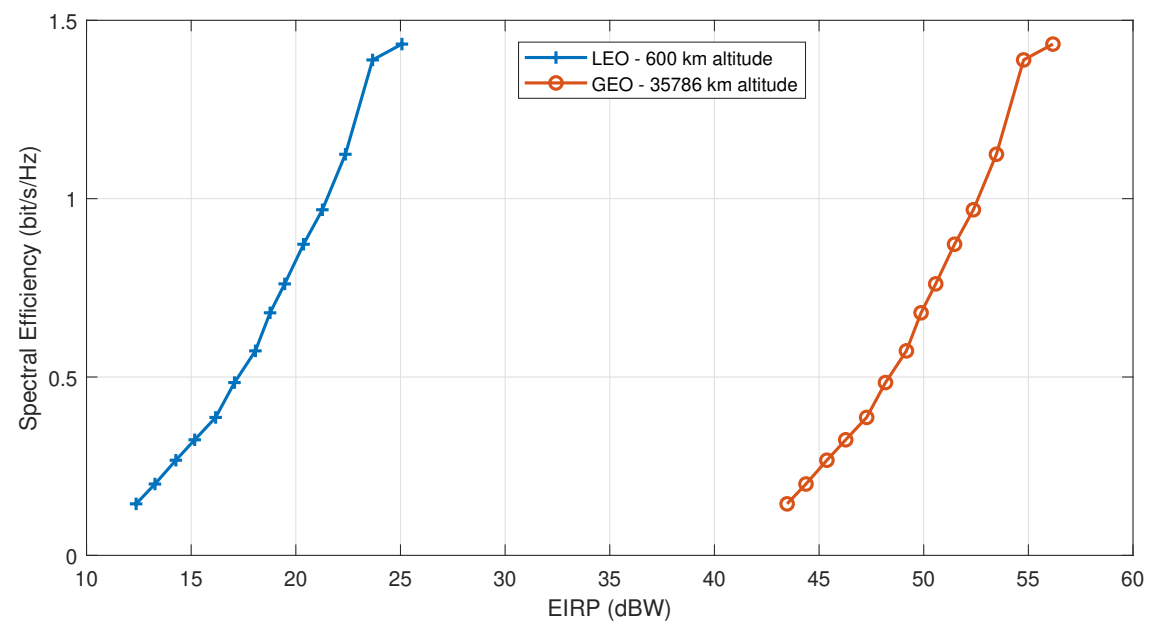

Fig. 2. Link budget result for downlink transmission.

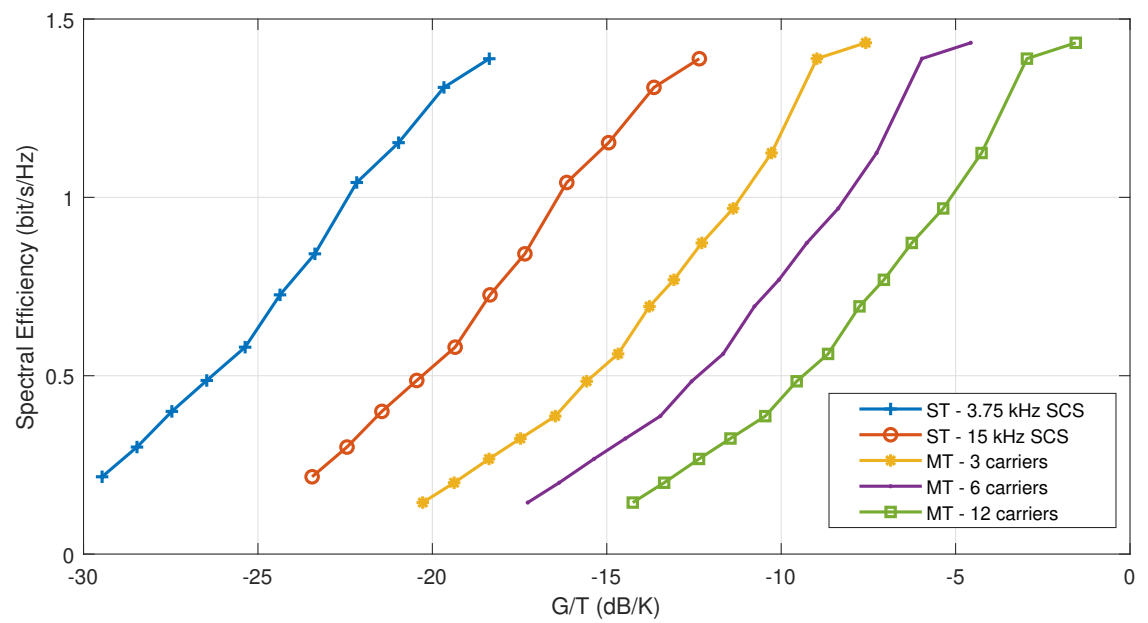

Fig. 3. Link budget result for uplink transmission, LEO satellite.

\section{Link Budget Impact in System Design}

Choosing one transmission mode or another, or sacrificing the spectral efficiency for the sake of closing the communication link, will directly impact the scheduling of the uplink and downlink channels. Consequently, the whole NB-IoT system will be affected, including important aspects such as delay, capacity and energy consumption. In this section, we will treat each of them separately, outlining 
To be presented in the 18th International Conference on Ad Hoc Networks and Wireless

(AdHoc-Now 2019), Luxembourg, 1-3 October 2019.

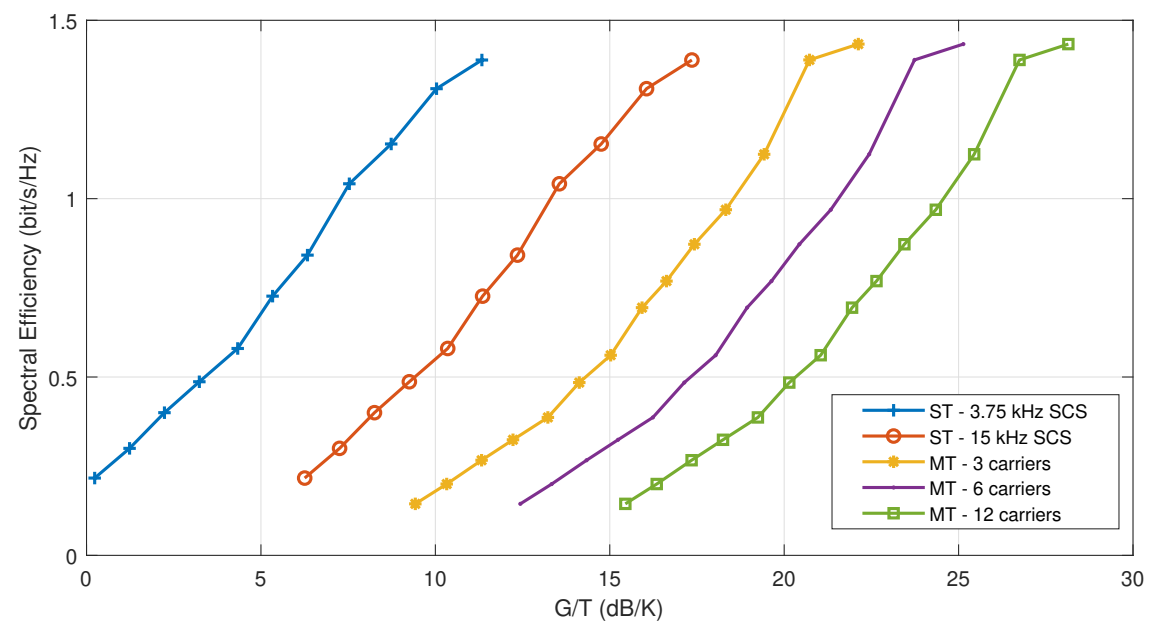

Fig. 4. Link budget result for uplink transmission, GEO satellite.

some system design trade-offs that should be considered when designing a nonterrestrial NB-IoT network.

\subsection{Delay}

In the downlink case, a lower spectral efficiency means that less useful data can be sent through NPDCCH, which is responsible for user scheduling. As a consequence, since we can send less useful information through this channel, the users have to wait for a longer time until they get all the necessary information to schedule their uplink transmission. As a matter of fact, this would cause a delay in the overall system. In the uplink transmission, being constraint of using less tones due to a lower $\mathrm{G} / \mathrm{T}$, would result in longer channels in time. Thus, the base station is forced to wait more time to receive a certain data packet by the user device.

\subsection{Capacity}

The capacity of the NB-IoT system has to do with the number of user devices that can access the network and be satisfied with service. The more frequent the NPRACH is sent in the uplink frame, the larger the probability that more devices can access the network. However, if we are constraint to use less resources for transmission in the frequency domain (less subcarriers) because of a low satellite $\mathrm{G} / \mathrm{T}$, less frequent the NPRACH can be sent since the radio frame would be occupied by the long NPUSCH in time of other users. This would significantly limit the number of devices that can access the network. 
To be presented in the 18th International Conference on Ad Hoc Networks and Wireless

(AdHoc-Now 2019), Luxembourg, 1-3 October 2019.

\subsection{Energy Consumption}

As we already emphasized in the introduction, the extended battery life is a very important feature of the NB-IoT technology. For this reason, the more often the devices fall into deep sleep mode, the more battery can be saved. However, this would require very short transmissions in time, which in our NB-IoT over satellite scenario can be impossible due to satellite power limitations. Again like already emphasized, closing the link by using less frequency resources or a lower MCS (lower spectral efficiency) is possible in such situations. However, this would translate in a longer transmission time interval (TTI) and less frequent deep sleep modes by the user device, thus more battery will be consumed.

\subsection{Other Considerations}

It is worth reminding here that the above-shown link budget results are for the BLER target of $10 \%$. By using the HARQ operation the link reliability would be improved because the same packed would be retransmitted if a NACK is received by the user or base station. Due to the presence of the satellite channel, the HARQ operation would cause a significant delay, which is much larger than the one experienced in a terrestrial network. Therefore, it has recently been discussed in the $3 \mathrm{GPP}$ the idea of deactivating the HARQ operation for NTN [2]. Doing this would require a BLER target adjustment (e.g. 1\% BLER), thus more EIRP and $\mathrm{G} / \mathrm{T}$ at the satellite for being able to close the communication link. Again, all the above-mentioned trade-offs should be considered in the system design.

\section{Conclusions}

In this paper, we studied the radio link budgets in order to support a reliable communication of IoT user devices with the corresponding base station in an NB-IoT over satellite system. The link and device parameters were chosen in accordance with the latest $3 \mathrm{GPP}$ specifications, while the satellite parameters were left open for design. The achievable spectral efficiency as a function of satellite antenna EIRP and G/T were shown through numerical simulations for both, LEO and GEO satellite, and under different transmission modes. It was shown that, in the downlink case, to enable an NB-IoT system capable of achieving the highest possible spectral efficiency, it is needed a minimum EIRP of $25 \mathrm{dBW}$ for a LEO satellite at $600 \mathrm{~km}$ altitude and $57 \mathrm{dBW}$ for a GEO satellite at $35786 \mathrm{~km}$ altitude. In the uplink, for a 12-carrier transmission mode it is required a minimum $\mathrm{G} / \mathrm{T}$ of $-2 \mathrm{~dB} / \mathrm{K}$ for a $\mathrm{LEO}$ satellite and $28 \mathrm{~dB} / \mathrm{K}$ for a GEO satellite. In case of lower values of $\mathrm{G} / \mathrm{T}$, the link can still be closed by using the other transmission modes (e.g. 1,3 or 6 subcarriers) for the SC-FDMA signal or sacrificing in spectral efficiency. Last but not least, the impact that different power budget would have in important features of NB-IoT technology, such as delay, capacity and power consumption, was discussed and analyzed. 
To be presented in the 18th International Conference on Ad Hoc Networks and Wireless (AdHoc-Now 2019), Luxembourg, 1-3 October 2019.

\section{Appendix: NB-IoT PHY layer simulation}

To derive the required SNR value for each MCS level assuring BLER target of $10^{-1}$, the NB-IoT PHY layer is implemented in Matlab and the performance in terms of BLER vs SNR is evaluated through numerical simulations. The baseband block diagram of the simulator is given in Figure 5 and the simulation parameters are summarized in Table 2. Overall, the following steps are performed for the BLER, SNR and spectral efficiency (SE) calculations:

- The bits are transmitted in block according to the transmission block size (TBS) given in the standard [7]. Changing the TBS would change the transmission code rate, hence enabling different performance gains for different MCS levels.

- The OFDM/SC-FDM baseband waveform generation follow the steps determined in the standard [6]. Please note that the N-point DFT/IDFT is applied only for SC-FDM waveform.

- The channel used is an additive white Gaussian noise (AWGN) channel.

- The receiver operations are performed and the erroneous TBS are counted. We run the simulations in order to guarantee at least 100 erroneous TBS for each SNR value.

- Obtaining the BLER-SNR curves, we find the minimum value of SNR that guarantees the BLER target of $10^{-1}$ for each MCS level. We repeat the simulation for downlink and uplink under different transmission modes.

- To calculate the spectral efficiency for each MCS level and transmission mode, the following formula is used:

$$
S E=\frac{T B S / T T I}{B W}(b i t / s / H z)
$$

where TTI is the transmission time interval corresponding to a certain TBS in each MCS level. Please note that in each MCS level we choose the TBS that gives the maximum throughput.

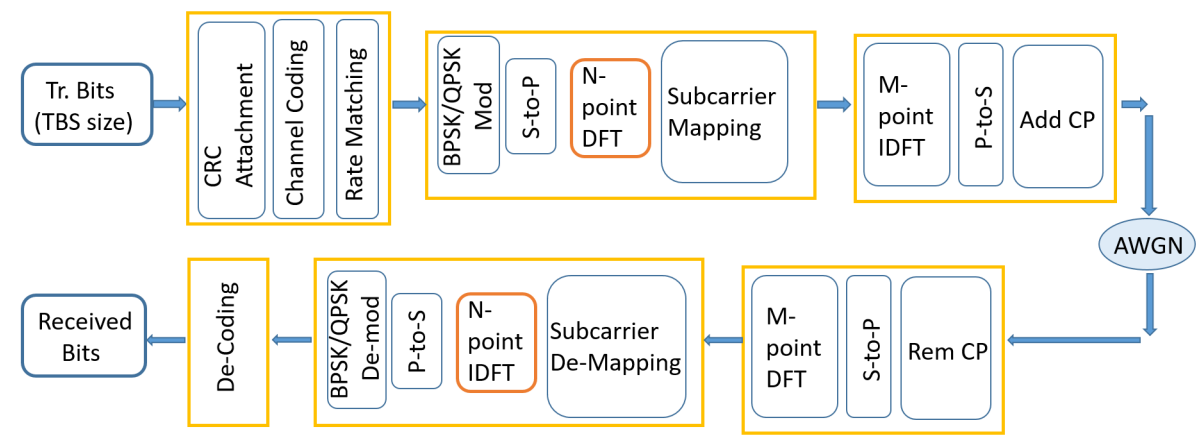

Fig. 5. Baseband Simulator Block Diagram 
To be presented in the 18th International Conference on Ad Hoc Networks and Wireless (AdHoc-Now 2019), Luxembourg, 1-3 October 2019.

- Please note that the performance of downlink and multi-tone uplink are almost the same (only different for some MCS). This is because table 16.5.1.2-1 and 16.5.1.2-1 from [7], containing the TBS value for each MCS, are almost identical.

Table 2. Simulation Parameters.

\begin{tabular}{|c|c|c|c|}
\hline Parameter & Uplink MT & Uplink ST & Downlink \\
\hline N FFT & 128 & 128 & 128 \\
Bandwidth & $(45,90,180) \mathrm{kHz}$ & $(3.75,15) \mathrm{kHz}$ & $180 \mathrm{kHz}$ \\
SCS (kHz) & $15 \mathrm{kHz}$ & $(3.75,15) \mathrm{kHz}$ & $15 \mathrm{kHz}$ \\
Modulation Format & SC-FDM & SC-FDM & OFDM \\
Modulation Order & QPSK & BPSK, QPSK & QPSK \\
MCS Selection & Table 16.5.1.2-2[7] & Table 16.5.1.2-2[7] & Table 16.4.1.5.1-1[7] \\
Coding Scheme & Turbo Code & Turbo Code & Turbo Code \\
CRC Bits & 24 & 24 & 24 \\
MCS range & $0-13$ & $0-10$ & $0-13$ \\
Channel & AWGN & AWGN & AWGN \\
\hline
\end{tabular}

Table 3. Simulation Results.

\begin{tabular}{|c|c|c|c|c|c|c|}
\hline & Uplink MT & \multicolumn{2}{c|}{ Uplink ST } & \multicolumn{2}{c|}{ Downlink } \\
\hline MCS & $S N R(d B)$ & SE (bit/s/Hz) & $S N R(d B)$ & SE (bit/s/Hz) & $S N R(d B)$ & SE (bit/s/Hz) \\
\hline 0 & -5.8 & 0.1444 & -4.2 & 0.2167 & -5.8 & 0.1444 \\
1 & -4.9 & 0.2 & -3.2 & 0.3 & -4.9 & 0.2 \\
2 & -3.9 & 0.2667 & -2.2 & 0.4 & -3.9 & 0.2667 \\
3 & -3 & 0.324 & -1.2 & 0.4867 & -3 & 0.324 \\
4 & -2 & 0.3867 & -0.1 & 0.58 & -2 & 0.3867 \\
5 & -1.1 & 0.4844 & 0.9 & 0.7267 & -1.1 & 0.4844 \\
6 & -0.2 & 0.5611 & 1.9 & 0.8417 & -0.1 & 0.5733 \\
7 & 0.7 & 0.6944 & 3.1 & 1.0417 & 0.6 & 0.68 \\
8 & 1.4 & 0.7689 & 4.3 & 1.1533 & 1.3 & 0.7611 \\
9 & 2.2 & 0.8722 & 5.6 & 1.3083 & 2.2 & 0.8722 \\
10 & 3.1 & 0.9689 & 6.9 & 1.3887 & 3.1 & 0.9689 \\
11 & 4.2 & 1.1244 & & & 4.2 & 1.1244 \\
12 & 5.5 & 1.3889 & & & 5.5 & 1.3889 \\
13 & 6.9 & 1.4333 & & & 6.9 & 1.4333 \\
\hline
\end{tabular}


To be presented in the 18th International Conference on Ad Hoc Networks and Wireless (AdHoc-Now 2019), Luxembourg, 1-3 October 2019.

\section{References}

1. 3GPP: LTE; Evolved Universal Terrestrial Radio Access (E-UTRA); User Equipment (UE) radio transmission and reception (Release 14). Technical Specification (TS) 36.101, 3rd Generation Partnership Project (3GPP) (04-2017)

2. 3GPP: Discussion on HARQ for NTN, . TSG RAN WG1 Meeting nr. 96bis, Xian, China, R1-1904859, 3rd Generation Partnership Project (3GPP) (04-2019)

3. 3GPP: Discussion on link budget for NTN, . TSG RAN WG1 Meeting nr. 96bis, Xian, China, R1-1903998, 3rd Generation Partnership Project (3GPP) (04-2019)

4. 3GPP: Technical Specification Group Radio Access Network; Study on New Radio (NR) to support non terrestrial networks; (Release 15). Technical Report (TR) 38.811, 3rd Generation Partnership Project (3GPP) (06-2018), version 15.0.0

5. 3GPP: LTE; Evolved Universal Terrestrial Radio Access (E-UTRA); Radio Resource Control (RRC); Protocol Specification; (Release 13). Technical Specification (TS) 36.331, 3rd Generation Partnership Project (3GPP) (08-2016), version 13.2.0

6. 3GPP: LTE; Evolved Universal Terrestrial Radio Access (E-UTRA); Multiplexing and channel coding; Version 15.3.0; (Release 15). Technical Specification (TS) 36.212, 3rd Generation Partnership Project (3GPP) (10-2018), version 15.3.0

7. 3GPP: LTE; Evolved Universal Terrestrial Radio Access (E-UTRA); Physical layer procedures; (Release 14). Technical Specification (TS) 36.213, 3rd Generation Partnership Project (3GPP) (10-2018), version 14.7.0

8. 3GPP: Cellular system support for ultra-low complexity and low throughput Internet of Things (CIoT) (Release 13) . Technical Report (TR) 45.820, 3rd Generation Partnership Project (3GPP) (11-2015)

9. Al-Fuqaha, A., Guizani, M., Mohammadi, M., Aledhari, M., Ayyash, M.: Internet of Things: A Survey on Enabling Technologies, Protocols, and Applications. IEEE Communications Surveys Tutorials 17(4), 2347-2376 (Fourthquarter 2015). https://doi.org/10.1109/COMST.2015.2444095

10. Alagha, N.: Satellite Air Interface Evolutions in the $5 \mathrm{G}$ and IoT Era. SIGMETRICS Perform. Eval. Rev. 46(3), 93-95 (Jan 2019). https://doi.org/10.1145/3308897.3308941, http://doi.acm.org/10.1145/3308897.3308941

11. Cioni, S., De Gaudenzi, R., Del Rio Herrero, O., Girault, N.: On the Satellite Role in the Era of 5G Massive Machine Type Communications. IEEE Network 32(5), 54-61 (Sep 2018). https://doi.org/10.1109/MNET.2018.1800024

12. CISCO white paper: Cisco visual networking index: Global mobile data traffic forecast update, 2015-2020

13. Cluzel, S., Franck, L., Radzik, J., Cazalens, S., Dervin, M., Baudoin, C., Dragomirescu, D.: 3gpp nb-iot coverage extension using leo satellites. In: 2018 IEEE 87th Vehicular Technology Conference (VTC Spring). pp. 1-5 (June 2018). https://doi.org/10.1109/VTCSpring.2018.8417723

14. Gineste, M., Deleu, T., Cohen, M., Chuberre, N., Saravanan, V., Frascolla, V., Mueck, M., Strinati, E.C., Dutkiewicz, E.: Narrowband IoT Service Provision to 5G User Equipment via a Satellite Component. In: 2017 IEEE Globecom Workshops (GC Wkshps). pp. 1-4 (Dec 2017). https://doi.org/10.1109/GLOCOMW.2017.8269209

15. Kodheli, O., Andrenacci, S., Maturo, N., Chatzinotas, S., Zimmer, F.: Resource Allocation Approach for Differential Doppler Reduction in NB-IoT over LEO Satellite. In: 2018 9th Advanced Satellite Multimedia Systems Conference and the 15th Signal Processing for Space Communications Workshop (ASMS/SPSC). pp. 1-8 (Sep 2018). https://doi.org/10.1109/ASMS-SPSC.2018.8510724 
To be presented in the 18th International Conference on Ad Hoc Networks and Wireless (AdHoc-Now 2019), Luxembourg, 1-3 October 2019.

16. Kodheli, O., Andrenacci, S., Maturo, N., Chatzinotas, S., Zimmer, F.: An Uplink UE Group-Based Scheduling Technique for 5G mMTC Systems Over LEO Satellite. IEEE Access 7, 67413-67427 (2019). https://doi.org/10.1109/ACCESS.2019.2918581

17. Kovács, I.Z., Mogensen, P., Lauridsen, M., Jacobsen, T., Bakowski, K., Larsen, P., Mangalvedhe, N., Ratasuk, R.: Lte iot link budget and coverage performance in practical deployments. In: 2017 IEEE 28th Annual International Symposium on Personal, Indoor, and Mobile Radio Communications (PIMRC). pp. 1-6 (Oct 2017). https://doi.org/10.1109/PIMRC.2017.8292260

18. Palattella, M.R., Dohler, M., Grieco, A., Rizzo, G., Torsner, J., Engel, T., Ladid, L.: Internet of Things in the 5G Era: Enablers, Architecture, and Business Models. IEEE Journal on Selected Areas in Communications 34(3), 510-527 (March 2016). https://doi.org/10.1109/JSAC.2016.2525418

19. Ratasuk, R., Tan, J., Mangalvedhe, N., Ng, M.H., Ghosh, A.: Analysis of nb-iot deployment in lte guard-band. In: 2017 IEEE 85th Vehicular Technology Conference (VTC Spring). pp. 1-5 (June 2017). https://doi.org/10.1109/VTCSpring.2017.8108184

20. Ratasuk, R., Vejlgaard, B., Mangalvedhe, N., Ghosh, A.: Nb-iot system for $\mathrm{m} 2 \mathrm{~m}$ communication. In: 2016 IEEE Wireless Communications and Networking Conference Workshops (WCNCW). pp. 428-432 (April 2016). https://doi.org/10.1109/WCNCW.2016.7552737

21. Scalise, S., Niebla, C.P., De Gaudenzi, R., Del Rio Herrero, O., Finocchiaro, D., Arcidiacono, A.: S-MIM: a novel radio interface for efficient messaging services over satellite. IEEE Communications Magazine 51(3), 119-125 (March 2013). https://doi.org/10.1109/MCOM.2013.6476875

22. $\mathrm{Xu}, \mathrm{L} . \mathrm{D} ., \mathrm{He}, \mathrm{W} ., \mathrm{Li}, \mathrm{S} .:$ Internet of Things in Industries: A Survey. IEEE Transactions on Industrial Informatics 10(4), 2233-2243 (Nov 2014). https://doi.org/10.1109/TII.2014.2300753 